Itinéraires Itinéraires

Littérature, textes, cultures

2012-3 | 2013

Lire les villes marocaines

\title{
Le Tanger expérimental de William Burroughs
}

\section{Xavier Garnier}

\section{OpenEdition}

\section{Journals}

Édition électronique

URL : http://journals.openedition.org/itineraires/924

DOI : 10.4000/itineraires.924

ISSN : 2427-920X

Éditeur

Pléiade

\section{Édition imprimée}

Date de publication : 1 juillet 2013

Pagination : $35-45$

ISBN : 978-2-343-01183-7

ISSN : 2100-1340

\section{Référence électronique}

Xavier Garnier, «Le Tanger expérimental de William Burroughs », Itinéraires [En ligne], 2012-3 | 2013, mis en ligne le 01 décembre 2012, consulté le 29 septembre 2020. URL : http://

journals.openedition.org/itineraires/924; DOI : https://doi.org/10.4000/itineraires.924

Itinéraires est mis à disposition selon les termes de la licence Creative Commons Attribution - Pas d'Utilisation Commerciale - Pas de Modification 4.0 International. 


\title{
Le Tanger expérimental de William Burroughs
}

\begin{abstract}
It is in Tangier, town where was written Naked lunch, that William Burrroughs really became a writer. My hypothesis is that this town, with its specific topography and its status of international zone, induced a crystallization of the writing. Tangier is not only for Burroughs a town where homosexual prostitution and drugs traffic are easy, it is a place where a contact was established with obscure forces that the man who accidentally killed his own wife few years earlier, was seeking to encounter.
\end{abstract}

Keywords : William Burroughs, Tangier, labyrinth, drugs, globalization

Mots clés : William Burroughs, Tanger, labyrinthe, drogues, mondialisation

C'est à l'occasion de son séjour à Tanger, entre 1954 et 1957, et de l'écriture de ce qui deviendra Le Festin nu, que William Burroughs confirme sa vocation d'écrivain. Il a déjà pas mal écrit auparavant : une correspondance nourrie avec Allen Ginsberg, deux romans Junkie (1953) et Queer (qui restera longtemps non publié), mais il ne se considère pour autant pas comme un écrivain. Il écrira en 1985 dans son introduction à Queer que l'événement déclencheur de l'écriture a été la mort accidentelle de sa femme, tuée de sa propre main. Cette mort ne lui laissera « d'autre choix que celui d'écrire, et de s'affranchir en écrivant ${ }^{1} »$. Il va se passer à Tanger comme une cristallisation de son écriture. Ceux qui, comme Paul Bowles, ont connu Burroughs à Tanger témoignent de la frénésie d'écriture qui l'a saisi lors de son séjour : "Le fatras sur son bureau et, en dessous, sur le sol, était chaotique; mais il ne consistait que dans des pages du Festin $n u$ auquel il travaillait sans relâche ${ }^{2}$. » Voici ce qu'il écrit à Ginsberg le 20 décembre 1956: "Je t'enverrai environ cent pages d'Interzone, ça

1. William Burroughs, Queer [1985], trad. Sylvie Durastanti, Paris, Christian Bourgois, 1986, p. 25.

2. Cité par Gérard-Georges Lemaire dans sa préface à la traduction française d'Interzone, Paris, Christian Bourgois, 1991, p. 13. 
sort tellement vite que j'ai à peine le temps de tout taper et ça me secoue comme un grand vent noir dans les os ${ }^{3} . »$

Mon hypothèse est que Tanger, en tant que lieu, est un agent déterminant de cette cristallisation qui donnera lieu à l'écriture du Festin nu. Quand on lit la correspondance de Burroughs, on se rend compte à quel point l'endroit où il vit est une donnée capitale de l'expérience qu'il est en train de vivre. Dans une lettre à Ginsberg datée du 13 octobre 1956, il évoque une relation quasi mystique avec cette ville :

Je suis entré dans une période de changements plus profonds que l'adolescence ou la prime enfance. Je vis en permanence dans mes numéros. Cela va si loin qu'un jour, je vais dépasser le point de non-retour. Je n'ai pas le temps de t'expliquer toutes les expériences mystiques que je vis dès que je franchis le seuil de la porte. Il y a quelque chose de spécial à Tanger. C'est le seul endroit où quand j'y suis, je ne veux être nulle part ailleurs. Ici pas de crise d'angoisse. Cette ville est belle car elle ne cesse de changer et de se recombiner. Venise est magnifique, mais elle ne change jamais. C'est un rêve figé dans la pierre. Et c'est le rêve de quelqu'un d'autre. L'effet suprême m'est cauchemardesque - Exemple : ciel supersonique, bleu orgone, vent chaud, un escalier en pierre conduisant à la Vieille Ville. En descendant les escaliers, un garçon arabe très sombre de peau avec une chemise mauve ${ }^{4}$.

La référence à Venise est inspirée par le motif du labyrinthe qui est central dans l'usage initiatique que Burroughs fera de Tanger. Le labyrinthe tangérois a des caractéristiques propres, que Burroughs va élucider à travers l'effet que cette ville provoque sur lui. Écrire la ville c'est écrire l'expérience que l'on fait de la ville, et cette expérience ne saurait être véritable si elle ne provoque pas un bouleversement des données de la subjectivité. Burroughs ne propose donc, dans le Festin nu, ni une description objective ni une lecture subjective de Tanger, mais rend compte d'une expérience mystique, provoquée par un dispositif particulier à cette ville.

\section{De la zone internationale à l'Interzone}

Il a souvent été remarqué que Burroughs n'a aucun goût pour l'exotisme, ou plus largement pour l'idée d'une rencontre avec d'autres cultures. L'idée d'altérité culturelle n'exerce sur lui aucun attrait, il y est même hostile : « Ici l'Orient rencontre l'Occident en une débâcle de totale incompréhension, chacun attendant de l'Autre la Réponse, le Secret, sans pouvoir la trouver, car ni l'un ni l'autre n'en détiennent ${ }^{5}$. ") Tanger ne sera donc certainement pas pour Burroughs un lieu de rencontres interculturelles, mais plutôt le lieu d'une débâcle, qui signe l'échec de l'approche culturaliste

3. William S. Burroughs, Lettres [The Letters of William S. Burroughs (1945-1959), 1993], Paris, Christian Bourgois, 2007, p. 501.

4. Ibid., p. 478.

5. William S. Burroughs, Interzone [1989], Paris, Christian Bourgois, 1991, p. 126. 
de la réalité. Dans la vision anarchisante de Burroughs, les identités culturelles sont toujours fabriquées par des officines de nature bureaucratique, qui s'en servent de façon stratégique. En ce sens, ses propos sur le fiasco de la rencontre entre l'Orient et l'Occident rejoignent les réflexions d'Edward Said sur un fantasme réciproque catastrophique. L'Orientalisme de Said peut être lu comme l'histoire de la fabrication de l'Orient par une « officine» occidentale, puis de sa reprise par des « officines » orientales. Ce que Burroughs va trouver à Tanger, c'est un espace interstitiel entre les « officines » culturelles.

Le terme d'interzone, qu'il utilise pour nommer l'espace tangérois, et qui est dérivé du statut officiel de "zone internationale ", intéresse Burroughs pour son préfixe. Dans l'Interzone, ce qui compte sont les interstices entre les lieux identifiés et culturellement assignés. Comme il l'explique dans un texte très virulent du Festin nu contre la bureaucratie : " Il y a toujours un interstice entre les officines ${ }^{6}$. " Dans cette ville prise en main par quatre administrations et divisée en secteurs américain, français, espagnol et anglais, la bureaucratie ne contrôle pas grand-chose et les espaces interstitiels de non-droit prolifèrent. Le mépris que Burroughs exprime pour l'altérité culturelle est exactement le même que celui qu'il manifeste pour ce qui serait censé être sa propre culture et ce qu'il appelle « la pourriture fondamentale de l'Amérique ». L'anarchisme de Burroughs le rend particulièrement agressif à l'égard de toute forme d'expression d'une homogénéité culturelle. L'idée même d'une identité culturelle, fabriquée dans des officines, est pour lui une affaire de bureaucrates.

Tanger n'est donc selon lui ni une ville arabe, ni une ville occidentale, mais une ville née dans l'entre-deux, fruit de l'impossible rencontre entre de multiples fantômes culturels. Le modèle que Burroughs a en tête, avant même d'arriver à Tanger, est celui du « camp de travail » ou de la « colonie pénitentiaire ». Tanger va être immédiatement perçue d'abord comme un camp de réfugiés où échouent les parias rejetés par leur propre monde : « Il y a ici une foule de gens incapables de partir, faute de papiers, d'argent, ou les deux. Tanger est une immense colonie pénitentiaire 7 . » Dans la biographie qu'il consacre à Burroughs, Ted Morgan fait remarquer que Tanger, construite à l'emplacement où fut enterré Antée, le titan vaincu par Hercule, est pour cet écrivain la ville des perdants, ou des $\operatorname{losers}^{8}$. Cet intérêt de Burroughs pour les villes-camps précède son arrivée à Tanger. On trouvera dès l'époque de la rédaction de Queer (en 1953), dans un texte intitulé « Rêve de la colonie pénitentiaire », un développement sur Quito qui annonce le Tanger du Festin nu: « Les détenus se mêlent aux gens du cru,

6. William S. Burroughs, Le Festin nu [Naked Lunch, 1959], Paris, Gallimard, 1964, p. 148.

7. William S. Burroughs, Interzone, op. cit., p. 119.

8. Ted Morgan, Literary outlaw. The Life and Time of William S. Burroughs [1988], Londres, Pimlico, 1991. 
dont il est difficile de les distinguer. Mais tôt ou tard, ils se trahissent par une intensité sortant de l'ordinaire, découlant du fait de se préoccuper exclusivement de s'enfuir' ${ }^{9}$. On n'habite pas ce genre de villes, on y échoue, et cela est valable tout autant pour les étrangers que pour les " gens du cru». Les Arabes eux-mêmes sont des marginaux qui vivent du contact avec les Européens : mendiants, arnaqueurs, guides véreux, entremetteurs, etc. La ville recycle ses habitants dans un immense espace de trafics en tout genre.

Or c'est précisément ce genre de villes que Burroughs choisit, non pour y habiter, mais pour y vivre. Il insiste dans ses lettres à Ginsberg sur le fait que Tanger est le seul lieu qui lui convienne et qui lui permette d'échapper à ses angoisses. Dans le film qu'il a fait à partir du Festin nu, David Cronenberg met directement en relation le séjour de Burroughs à Tanger et le meurtre accidentel de sa femme Joan : l'Interzone est un espace d'expiation. On comprend mieux dans cette perspective le rapport très particulier que Burroughs entretient avec la ville : il y vient pour passer un temps de purgation. Pour lui, comme pour tous ceux qu'il rencontre, Tanger est un lieu de transit, un camp où l'on attend l'heure de partir. Là est sans doute l'origine du différend latent avec Paul Bowles, qui se pensait installé à Tanger et voulait jouer le jeu de l'adaptation. Si l'exotisme naît du désir d'adopter l'autre culture, on comprend qu'il ne peut être la ligne d'écriture de Burroughs.

\section{L'envers du décor exotique}

La ville dans sa totalité est du coup une sorte d'envers du décor. Elle est perçue du point de vue de ses lieux les plus identitairement désinvestis. Voilà pourquoi les descriptions de l'Interzone peuvent renvoyer tout aussi bien à Quito, à New York, qu'à Tanger ou à toute autre ville que Burroughs connectera à l'Interzone. Les arrière-cours, les hangars désaffectés, les terrains vagues, sont autant de lieux qui rapprochent les villes à travers le monde. Ces lieux sont une première préfiguration de l'Interzone. C'est d'abord par ce type de lieux qu'une ville comme Tanger sera appréhendée.

Ces lieux oubliés, peu fréquentés par les humains, forment une sorte de doublure invisible de la ville, prête à en accueillir les cris et les rumeurs. Un parcours descriptif de l'Interzone se fait à l'occasion d'un cri lancé à travers la ville, dont le texte va s'attacher à suivre le parcours sonore :

Le cri jaillit de sa chair, traversa un désert de vestiaires et de dortoirs à soldats, et l'air moisissant de pensions saisonnières, et les couloirs spectraux de sanatoriums de montagne, l'odeur d'arrière-cuisine grise et grognonne et graillonnante des asiles de nuit et des hospices de vieillards, l'immensité poussiéreuse de hangars anonymes et d'entrepôts de douanes - traversa des portiques en ruine et des volutes de plâtre barbouillé, des pissoirs au zinc

9. William S. Burroughs, Interzone, op. cit., p. 97. 
corrodé en une dentelle transparente par l'urine de millions de lopettes, des latrines abandonnées aux mauvaises herbes et exhalant des miasmes de merde retournant en poussière, des champs de totems phalliques dressés sur la tombe des nations moribondes dans un bruissement plaintif de feuilles sous le vent - traversa encore le grand fleuve aux eaux boueuses où flottent des arbres aux branches chargées de serpents verts, et de l'autre côté de la plaine, très loin, des lémuriens aux yeux tristes contemplent les rives, et on entend dans l'air torride le froissement de feuilles mortes des ailes de vautours... Le chemin est jonché de préservatifs crevés et d'ampoules d'héroïne vides et de tubes de vaseline aplatis, aussi secs que l'engrais d'os sous le soleil d'été ${ }^{10} \ldots$

Saisissant portrait apocalyptique de l'Interzone, comme espace à proprement parler inhabitable mais qui dit une vérité de Tanger, telle que cette ville est expérimentée par Burroughs : un espace où l'envers du décor remonte à la surface.

Ces lieux désolés sont désespérants car inhabitables. Ils sont clairement pour Burroughs du côté de la mort et de l'apocalypse. On ne peut les traverser que poussé par un « grand vent noir » qui s'infiltre partout. Nous ne sommes jamais à l'intérieur de la ville, mais plutôt dans un dédale de ruelles ouvert sur le Dehors. Ce motif du vent glacial qui balaie la ville et s'engouffre dans les rues était déjà présent dans les textes de Queer sur Quito :

Cette nuit-là, Lee se vit en camp de travail. Alentour se dressaient de hautes montagnes pelées. Il vivait dans une pension perpétuellement glaciale. Il sortit se promener. Comme il tournait le coin d'une rue pour s'engager dans une autre, pavée et sale, un froid vent de montagne le frappa de plein fouet. Il se sangla dans sa canadienne, en se sentant saisi d'un désespoir infini ${ }^{11}$.

Ils vont être repris à propos de Tanger moyennant quelques aménagements. Le vent froid ne descendra plus des montagnes, mais viendra de la mer glaciale qui borde la ville. Burroughs voit la médina comme un grand labyrinthe ouvert sur la mer. Le vent froid venu de la mer et du large pénètre dans les ruelles et se propage dans tous les recoins de la ville, jusque dans les pièces les plus reculées des maisons. Aucun espace d'intériorité n'est ménagé dans la ville : «Nul dans la Cité ne peut verrouiller sa porte et quiconque le veut peut entrer n'importe où et n'importe quand ${ }^{12}$. » L'architecture globale de la ville obéit à ce principe d'extériorité :

Toutes les rues de la ville courent entre des gorges profondes qui débouchent sur une grande place en forme de haricot ${ }^{13}$. Les façades

10. William S. Burroughs, Le Festin nu, op. cit., p. 62-63.

11. William S. Burroughs, Queer, op. cit., p. 108.

12. William S. Burroughs, Le Festin nu, op. cit., p. 116.

13. On reconnaît à ce détail le Socco Chico. 
entourant la place constamment baignée d'ombre sont percées d'alvéoles, taudis ou cafés, les uns profonds de quelques pieds, les autres se perdant en un labyrinthe de chambres et de couloirs ${ }^{14}$.

La ville est architecturalement conçue pour laisser le « vent noir» s'infiltrer dans tous les interstices et faire éclater les intériorités. Le labyrinthe tangérois est un espace d'extériorité : le véritable problème n'est pas de savoir comment on en sort, mais s'il est possible de s'y aménager un espace intérieur.

En ouvrant le labyrinthe sur la mer, en ne présentant pas la médina comme un espace clos, Burroughs déjoue le piège exotique si souvent à l'œuvre dans les textes de l'époque coloniale sur les villes marocaines. La médina n'est pas le symbole de l'intériorité mystérieuse du monde arabe, elle est au contraire un dispositif d'extériorisation branché sur l'immensité du monde. En elle le monde s'engouffre et vient irriguer ses moindres alvéoles. La médina est ouverte à tous les flux, et en particulier les flux d'argent. La zone internationale est également une zone franche dans laquelle tous les trafics sont permis. Le centre de la ville sera le Socco Chico, que Burroughs présente comme l'épicentre, ou la «plaque tournante», de la ville.

\section{À chacun son « numéro »}

Les personnages ne peuvent habiter un tel espace évidé culturellement sans voir se modifier leurs comportements et leurs interactions. Burroughs insiste beaucoup sur le fait qu'à Tanger, chacun est pris dans un «numéro ». L'idée de «numéro » forme un très intéressant principe de construction de ses personnages, qui entre en relation avec la présentation d'un espace interstitiel. Parce que ceux qui ont échoué à Tanger sont des épaves et n'ont d'autre centre de gravité que le temps ouvert de l'attente, ils doivent se construire une façade pour éviter la désintégration de leur être. La ville distribue des rôles, qui sont autant de simulacres ou de postures par lesquelles les individus n'entrent pas en communication mais en contact. C'est du point de vue du personnage-numéro, et de la recherche du « contact», que se comprend la saisissante description du Socco Chico insérée dans un texte sur Tanger écrit pour raisons alimentaires et que Burroughs envoie à Ginsberg dans l'espoir qu'il le fasse publier dans un magazine ${ }^{15}$ :

Le Socco Chico est le lieu de rencontre, l'artère principale, la plaque tournante de Tanger. Tous les gens qui se trouvent en ville s'y montrent au moins une fois par jour. La plupart des résidents y passent l'essentiel de leur journée. De toutes parts, on peut voir des individus qui ont échoué là en dernier ressort, à bout de ressources, attendant un boulot, un virement, un visa, un permis de

14. William S. Burroughs, Le Festin nu, op. cit., p. 66.

15. Il s'agit du texte intitulé «Zone internationale », repris dans Interzone. 
séjour qu'ils n'auront jamais. Toute leur vie durant, ils n'ont pas eu de veine, prenant toujours le mauvais parti. Et c'est là qu'ils se retrouvent. En tout dernier ressort. Dernière escale : le Socco Chico de Tanger.

Le marché des échanges psychiques est tout aussi engorgé que les boutiques. Une cauchemardesque impression de paralysie sature le Socco, l'impression que rien ne peut survenir, que rien ne peut évoluer. Les conversations s'émiettent en inanités cosmiques. Les gens restent assis aux terrasses des cafés, aussi silencieux et isolés que des pierres. Tout autre rapport que de proximité physique est exclu. En l'absence de toute intervention humaine, l'évolution des lois de l'économie n'aboutit qu'à des équations de paralysie absolue. Un de ces jours, les jeunes Espagnols en imper passionnés de foot, les guides et arnaqueurs arabes glanant quelques sous et fumant leurs pipes de kif, les pédés assis aux terrasses des cafés pour reluquer les gamins, les gamins paradant sous leurs yeux, les tapeurs, les macs, les contrebandiers et les changeurs se pétrifieront à jamais en une ultime et absurde posture ${ }^{16}$.

Dans cet espace désorienté au cœur du labyrinthe, aucune communication n'est possible et chacun est enfermé dans son numéro, et pourtant chacun est à la recherche du contact. Même le rôle de l'exilé, de celui qui attend à Tanger une opportunité pour s'enfuir, est un «numéro » qui peut servir à taper de l'argent. Le ressort de l'évocation de Tanger par Burroughs passe par cette apparente contradiction entre une ville présentée comme en perpétuel mouvement (à la différence de Venise qui serait figée dans la pierre), et la pétrification des postures de ses occupants. Il y a là un aspect clé de la perception de Tanger comme « interzone », c'est-à-dire comme espace interstitiel entre les zones culturelles. Dans cet espace mouvant, aucune communication interpersonnelle n'est possible, faute de l'existence d'un socle commun. Burroughs raconte un monde livré à l'autisme. Les postures absurdes, plus ou moins excentriques, qu'adoptent les résidents de Tanger, relèvent d'une esthétique maniériste : les corps ne disent rien par eux-mêmes, ils s'organisent en un étrange ballet qui met en contact les figures sans permettre de véritables rencontres. Parce que chacun est menacé d'un effondrement intérieur, d'une « consternante désintégration », le numéro sert à se reconstituer par l'extérieur, par le contact avec un public.

À cette explication existentielle du «numéro » vient s'ajouter une explication économique : les personnages cherchent désespérément un public pour établir un contact de nature commerciale. Les numéros sont un effet direct des lois de l'économie. Pour que les flux d'argent puissent circuler, il faut bien que les contacts s'établissent entre l'offre et la demande. Le « personnage-numéro », comme leurre, est l'opérateur de cette mise en contact. Dans l'univers tangérois de Burroughs, la bonne question à se poser dès lors qu'un nouveau personnage apparaît sur la scène est de savoir ce qu'il cherche à « fourguer ». Le personnage est l'habillage humain d'une transaction ou d'un trafic qui cherche à se faire. Il y a là une modalité

16. William S. Burroughs, Interzone, op. cit., p. 105. 
d'existence du personnage très intéressante pour la théorie narrative : le personnage est l'opérateur fixe de la circulation des flux au sein d'un espace mouvant. La pétrification du personnage dans son rôle est la condition de circulation des flux. Dans ces trafics en tous genres qui traversent 1'espace tangérois, il en est un qui est pour Burroughs le paradigme de tous les autres : le trafic de drogue.

Une évocation new-yorkaise du trafic de drogue nous permet de mieux comprendre l'importance du personnage comme « contact » :

On voit parfois jusqu'à cinquante camés loqueteux et piaulant de souffrance filer au train d'un môme qui joue de l'harmonica, et soudain ils tombent sur le Type - le Contact, la Connection, le Fourgueur de Came, le vrai Camelot - le cul posé sur une canne-siège en train de jeter du pain aux cygnes, ou bien il apparaît sous les traits d'une tantouse en travesti qui balade son afghan du côté de la $50^{\mathrm{e}}$ Rue, ou d'un vieux pochard pissotant contre un pilier du métro aérien, ou d'un étudiant juif qui distribue des tracts socialisants à Washington Square, ou d'un forestier, ou d'un exterminateur de parasites - ou d'un chef de publicité pédouillard qui s'en jette un au snack Nadick et appelle le serveur par son prénom ${ }^{17} \ldots$

Les différentes apparences du dealer sont autant de «numéros », de leurres, de masques identitaires exhibés, qui dissimulent la seule réalité qui compte : l'accès à la «Came ». Derrière la façade de passants désœuvrés, la ville est livrée aux trafics en tous genres. La circulation de la drogue est le principe de connexion entre toutes les classes, toutes les races, tous les milieux. Les personnages différenciés qui hantent l'Interzone sont les formes qui rendent visible le flux invisible du trafic. D'où le caractère hallucinatoire de cette ville dont les formes ne se manifestent que pour permettre des mouvements invisibles. Tous les acteurs de Tanger sont des agents au service de forces invisibles, ils sont connectés à des réseaux mondiaux qui emportent la ville.

\section{Tanger : ville de « basse intensité » au cœur d'un réseau mondial}

Les biographes de Burroughs n'ont pas tort de rappeler que Tanger a permis à l'écrivain de satisfaire facilement ses penchants pour 1'homosexualité et pour la drogue. La prostitution masculine et l'accès simple aux stupéfiants, et en particulier à l'héroïne, ne sont certainement pas étrangers à son goût pour la ville. Or, Burroughs insiste sur le fait que les effets du sexe et de l'héroïne sont contradictoires, voire antagoniques. Alors que le sexe tend à une fusion des corps, par liquéfaction d'un corps dans un autre, l'héroïne pétrifie le corps du «camé » et l'isole émotionnellement du monde. On retrouve deux devenirs possibles du personnage. Soit les figures se heurtent et à ce moment, comme des bulles de savon, elles se fondent

17. William S. Burroughs, Le Festin nu, op. cit., p. 18. 
l'une dans l'autre; soit elles s'esquivent soigneusement pour conserver leur autonomie. Fusion des corps ou pétrification autiste : aucun type de communication intermédiaire ne semble possible. Les modalités du contact ne sont pas les mêmes selon que l'on bascule du côté du sexe, ou de la drogue. La parade homosexuelle n'obéit pas aux mêmes principes que la technique de vente du dealer. La consommation sexuelle est une affaire de lieux clos, en l'occurrence le bain turc, alors que la consommation de drogue concerne la ville dans son rapport avec le dehors.

On peut établir un lien entre la mer glaciale, traversée par un courant froid, qui borde Tanger et le « Grand froid » que recherchent les drogués, phénomène de «basse intensité » qui modifie leur rapport au temps. Le Grand Froid venu de la mer est comparable à celui que s'injecte le camé et qui doit circuler dans ses veines pour irriguer toutes les cellules du corps. La circulation du vent marin dans les ruelles de la médina, jusque dans les arrière-chambres des maisons, est en phase avec la circulation de la drogue dans les cellules du camé : c'est un principe de distribution de la «basse intensité ». C'est lorsqu'il évoque l'effet de l'héroöne que Burroughs rend compte de la façon la plus claire du type de rapport qu'il entretient avec la ville :

L'ingestion de drogue - et notamment d'héroïne lorsqu'elle est administrée à forte dose - provoque à plus ou moins long terme une dépression permanente du cerveau postérieur, très voisine de celle qu'on découvre au stade terminal de la schizophrénie : autisme, absence totale d'affect et quasitotale d'activité cérébrale. Le drogué peut rester face au mur sept ou huit heures d'affilée. Il a conscience de son environnement, mais celui-ci est dénué de toute implication émotive et, partant, d'intérêt ${ }^{18}$.

Cette neutralisation de l'émotivité qui caractérise la prise d'héroïne le met en phase avec une ville elle-même sans états d'âme, totalement livrée au marché. Mais le paradoxe est que cette ville, qui est une plaque tournante du marché mondial, n'est aucunement saisie de frénésie. Au contraire, Burroughs décrit de façon quasi clinique l'atonie qui frappe Tanger par saturation de marchandises :

Sous l'effet de cette constante circulation des denrées, la pénurie créée par l'état de guerre est comblée, les prix et les valeurs tendent à se stabiliser, et Tanger s'épuise comme l'univers soumis à l'entropie, où aucun mouvement n'est plus possible, dès l'instant où toute l'énergie est également distribuée ${ }^{19}$.

Le camé (tant qu'il n'est pas en «renonce») et la ville sont en phase dans une sorte d'ataraxie qui les place au cœur du monde. C'est par cette apathie, ce régime de basse intensité émotive, que Burroughs trouve un

18. Ibid., p. 49.

19. William S. Burroughs, Interzone, op. cit., p. 12. 
accord avec une population arabe en prise sur le majoun et le kif (dérivés du cannabis), drogues qui affectent le sentiment de l'écoulement du temps. Les seuls développements de type «culturaliste » qu'on pourra trouver sous la plume de Burroughs concernent cette délinéarisation de la structure temporelle dans le monde arabo-musulman. Ainsi de la musique arabe qui n'aurait « ni début, ni fin », et surtout du haschisch, « la drogue par excellence du monde islamique, [qui] affecte le sentiment de l'écoulement du temps de telle façon qu'au lieu d'être perçus dans la classique continuité du passé, du présent et de l'avenir, les événements apparaissent en simultanéité, l'instant présent renfermant à la fois le passé et l'avenir ${ }^{20} »$.

Dans sa quête d'un lieu d'expiation, Burroughs rend hommage à Tanger comme seul lieu qui ait su le placer dans l'œil du cyclone et ait libéré son écriture de la dictature du sens. Dans une lettre à Ginsberg, datée du 23 janvier 1957, dans laquelle il tente de convaincre son ami de venir lui rendre visite, il fait un «numéro » mystique qu'il faut prendre au sérieux en dépit de sa pirouette finale :

Interzone me vient comme sous la dictée, j'ai du mal à tenir le rythme. Je vais envoyer ce qui a été fait jusqu'à présent. Lis-le dans n'importe quel sens. Cela n'a aucune importance... Ma conversion religieuse est maintenant terminée. Je ne suis ni musulman ni chrétien, mais j'ai une grosse dette envers l'islam et n'aurais jamais pu entrer en contact avec Dieu AILLEURS QU'ICI. Je suis conscient de tout ce que j'ai pu absorber de cette religion en vivant en osmose avec elle et sans même parler un seul mot de cette langue effrayante. Je vais m'en occuper quand j'aurai un moment de temps libre. En ce moment, j'ai à peine le temps de manger et de baiser ${ }^{21} \ldots$

Or cette expérience mystique n'aurait pu se faire sans une prise en compte des perceptions concrètes de la ville telles qu'elles sont restituées dans ce texte qui aurait pu servir de présentation à l'ouvrage :

Ce livre expulse ses pages dans toutes les directions, kaléidoscope de panoramas divers, pot-pourri d'ariettes et de bruits de la rue, de vesses et de cris de guerre et de grincements de rideaux de fer dans les ruelles commerçantes - cris d'horreur et de passion, éthos et pathos et pataquès, miaulements du chat fornicateur et piaulements ultrasoniques du poissonchat déplacé, charabia prophétique du brujo dans les transes de la muscade, claquement de vertèbres des pendus, hurlements des mandragores, soupir de l'orgasme, silence de l'héroïne dans le silence en contrepoint des cellules assoiffées au petit matin, Radio Le Caire s'égosillant comme un commissaire-priseur pris de délire, les flûtes du Ramadan effleurant les nerfs malades du camé avec la fluide légèreté d'un détrousseur d'ivrognes tapi dans la grisaille du métro à l'aube et cherchant du bout des ongles le froissement vert du billet bouchonné... Voici la Prophétie et la Révélation ${ }^{22} \ldots$

20. Ibid., p. 119.

21. William S. Burroughs, Lettres, op. cit., p. 506.

22. William S. Burroughs, Le Festin nu, op. cit., p. 248-249. 
Ce livre de la « déprise » n'aurait sans doute pas été écrit si Burroughs n'avait pas rencontré sur sa route cette ville qui, au moins pour ce moment de son histoire, a réuni pour lui les conditions d'une expérience mystique du réel.

Xavier Garnier

Université Sorbonne Nouvelle, Sorbonne Paris Cité

Écritures de la modernité (EA 4400) 